\title{
Steroids and isoquercetin from Lygodium microphyllum
}

\author{
Hadi Kuncoro ${ }^{1 *}$, Kindi Farabi $^{2}$, Laode Rijai ${ }^{1}$ \\ ${ }^{1}$ Laboratory of Pharmaceuticals Research and Development, TROPICAL PHARMACA, Faculty of Pharmacy, Mulawarman University, Samarinda7 5119, \\ Kalimantan Timur, Indonesia. ${ }^{2}$ Departement of Chemistry, Faculty of Mathematics and Natural Sciences, Padjadjaran University, Jatinangor 45363, Sumedang, Jawa \\ Barat, Indonesia.
}

\begin{tabular}{|c|}
\hline ARTICLE INFO \\
\hline $\begin{array}{l}\text { Article history: } \\
\text { Received on: } 19 / 01 / 2017 \\
\text { Accepted on: 06/04/2017 } \\
\text { Available online: } 30 / 11 / 2017\end{array}$ \\
\hline 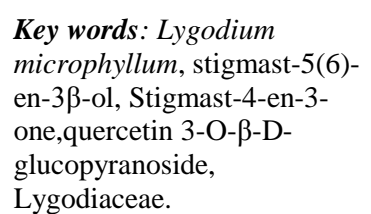 \\
\hline
\end{tabular}

\section{INTRODUCTION}

Fern has survived since Paleozoic era and can adapt to variety environmental changes (Wallace et al, 1991), thus fern has a lot of useful secondary metabolites, including flavonoids, steroids, alkaloids, phenols, triterpenoids, and various kinds of amino acids and fatty acids (Zeng-fu et al, 2008). One from thousands of species of ferns that have interesting pharmacological benefits is Lygodiaceae family. The only genus in this family is the genus Lygodium (Guo-gang et al, 2012). Generally, Lygodium genus is a group of ferns that spread and always propagate in other plants. Genus Lygodium are different from other kinds of ferns because it has roots that crawl on the

\footnotetext{
* Corresponding Author

Hadi Kuncoro, Laboratory Of Pharmaceuticals Research and Development, TROPICAL PHARMACA, Faculty Of Pharmacy, Mulawarman University, Samarinda7 5119, Kalimantan Timur, Indonesia. Email: kuncoro_hadi82 @ yahoo.com
}

ground rhizomes and fleshy and can only live in the open because they like the sunlight. Some plants from genus Lygodium are invasive and have become a problem in a number of forest areas. There are the fast growing plant and lack of predator makes them dominate, displacing wildlife, threatens biodiversity, and enhance the human-animal conflict. One of the invasive species from genus Lygodium is L. microphyllum. Plants of this genus have a variety of properties that have been widely recognized, thus the utilization of plants from genus is quite expected. Some herbs Lygodium widely used by people one of them as traditional medicine as hepatitis medicine (Zheng and Xing, 2009). Back pain, rheumatism and treatment for kidney stones (Lee et al, 2008), expectorant, scabies, eczema and liver treatment (Upreti et al, 2009), diuretics, antiplasmodial, and treatment for lung and kidney (Upreti et al, 2009), laxative, headache and digestive disorders (Zheng dan Xing, 2009), Menstrual painkiller, contraception, bone fracture and hemorrhoid treatment (Cambie and Ash, 1994) and cough treatment (Karthik, 2011). 
Phytochemical Studies on the genus Lygodium been reported to contain a compound with a structure that is unique and diverse biological activities such as flavonoids (Zhang et al, 2006), Glycoside phenolics (Ye et al, 2007), naphthoquinone (Chen et al, 2010), ecdysteroids (Zhuet al, 2009), phenylpropanoid glycoside (Duan et al, 2012).

L. microphyllum based on ethnobotany information and ethnopharmacological provides many benefits in the field of reproductive health as well as simple patterns make the plant is classified as herbs are easily available so that benefit of these plants is required primarily to determine the chemical constituents of this plant. In our ongoing research to find new compounds from Indonesia Lygodium plants, has been investigated Lygodium a chemical plant that grows in Borneo. In this communication, we will report the steroid compound, stigmast-5 (6) -en-3 $\beta$-ol and stigmast-4-en-3-one from the n-hexane extract and quercetin 3-O$\beta$-D-glucopyranoside from the extract ethyl acetate plant $L$. microphyllum

\section{MATERIALS AND METHOD}

Melting point measured on electrothermal melting point apparatus and not corrected. IR spectra were measured on PerkinElmer 1760X spectrophotometer, FT-IR on KBr. Massspectra recorded with a mass spectrometer Water, Qtof HR-MS XEV ${ }^{\mathrm{otm}}$. ${ }^{1} \mathrm{H}$ and ${ }^{13} \mathrm{C}$ NMR spectraare obtained by JEOL NMR $500 \mathrm{MHz}$, JEOL NMRECZR 600 Mhzused TMS as an internal standard. Chromatographic separation is carried out on silica gel 60 (Merck), ODS (Fuji silysia). TLC Plate filled with silica gel $\mathrm{GF}_{254}$ (Merck, $0.25 \mathrm{~mm}$ ) and detection was obtained with the appearance of $10 \% \quad \mathrm{H}_{2} \mathrm{SO}_{4}$ in ethanol followed by heating and under ultraviolet-visible light at wavelengths of 257 and $364 \mathrm{~nm}$. Preparative MPLC using a Buchi Pump Controller C-610, Buchi Pump Modules C-605 with FLH-R10030B SiliCycle columnISO04 Siliasep ${ }^{T M}$.

\section{Plant material}

L. microphyllum plant material is obtained from forest areas in Samarinda, East Kalimantan in June 2014. The plant is identified by staff at the Faculty of Forestry, University of Mulawarman, Samarinda and sample specimens stored at the Faculty of Forestry, University of Mulawarman, Samarinda.

\section{Extraction and isolation}

\section{Compound isolation from n-hexane extract}

Dried powder of L. microphyllum $(3.54 \mathrm{~kg})$ was extracted with methanol at room temperature. The ethanol extract obtained was concentrated at low pressure to produce methanol extract concentrated dark brown $(526 \mathrm{~g})$. The further concentrated methanol extract was dissolved in water (4: 1) and partitioned successively with n-hexane, ethyl acetate, and n-butanol. Evaporation of the solvent from the resulting extract each successive n-hexane extract (59 g), ethyl acetate (72 g) and nbutanol (54 g). Most of the n-hexane extract (50 g) were separated by vacuum liquid chromatography (VLC) on silica gel G60 with nhexane-ethyl acetate-methanol, 10\% increasing polarity generated 25 fractions (H01-25). Faction H3-11, combined (4 g) was separated by a column chromatography on silica gel (70-230 mesh) with the eluent n-hexane-ethyl acetate, $1 \%$ increased polarity generated 23 fractions (F01-23). F8 fraction separated using Medium performance liquid chromatography (MPLC) preparative with column Silicycle FLH-R10030B-ISO04 Siliasep $^{\mathrm{TM}}$ eluted by comparison isocratic solvent, chloroform: ethyl acetate (9: 1), the pump speed is $3 \mathrm{~mL} / \mathrm{min}$, accommodated every 30 seconds to 50 fractions and guided by TLC, fractions obtained 50 fractions divided into two namely D1 (1-5) and D2 (650), D2 subfraction separated using moderate performance liquid chromatography (MPLC) preparative with SiliCycle FLHR10030B-ISO04 Siliasep ${ }^{T M}$ column eluted by comparison isocratic solvent, toluene: chloroform (8:2), the pump speed of 1.5 $\mathrm{mL} / \mathrm{min}$, accommodated every 30 seconds into 30 fractions and guided by TLC, subfraction D2C (15-30) are separated again using Medium Performance Liquid Chromatography (MPLC) preparative with SiliCycle FLH-R10030B-ISO04 Siliasep ${ }^{T M}$ column eluted by comparison isocratic solvent, toluene: chloroform (8: 2), the pump speed of $1.2 \mathrm{~mL} / \mathrm{min}$, accommodated every 30 seconds into 30 fractions and guided by TLC, subfraction D2C2 (21-30) was purified using preparative thin layer chromatography eluted with a ratio of solvent, chloroform: ethyl acetate: acetic acid (9: 1: 0.5) and needle-shaped crystals obtained stigmast-4-en-3-one (5 mg). Fraction H10-16, combined (4.6 g) were separated by column chromatography on silica gel (70-230 mesh) with the eluent n-hexane-ethyl acetate, 5\% increasing polarity generated 25 fractions (I01-25). I012-17 fraction combined $(230 \mathrm{mg}$ ) were separated by preparative thin layer chromatography on silica gel $\mathrm{GF}_{254}$ with chloroform eluent: ethyl acetate: acetic acid (9: 1: 0.5 ) to produce as much as $40.6 \mathrm{mg}$ white solid that is subsequently crystallized with n-hexane-ethyl acetate produced white crystals colorless stigmast-5 (6) -en-3 $\beta$-ol (38 mg).

\section{Compound isolation from ethyl acetate extract}

The ethyl acetate extract was separated using column chromatography open with ODS eluted with a gradient solvent ratio, water: Methanol: Ethyl Acetate, guided by TLC, produced 22 fractions (1-22), Fraction 5-7 (B) were merged and acquired $920 \mathrm{mg}$, then separated using column chromatography on silica G60 open eluted with a gradient ratio of solvent, ethyl acetate: methanol, guided by TLC and obtained 22 fractions (B1-22), fraction B2 (B2-B5) merged and acquired $420 \mathrm{mg}$, then separated using open column chromatography on silica G60 eluted with a gradient ratio of solvent, ethyl acetate: methanol, guided by TLC to obtain 22 fractions (B2.1-22), fraction B2.2 (B2B) as much as $190 \mathrm{mg}$ open separated using column chromatography on silica G60 eluted with a gradient solvent ratio, chloroform: methanol, guided by TLC, and obtained 14 fractions (B2B.1-14), fractions to 9 (B2B.9) was further purified using preparative thin layer 
chromatography eluted with a ratio of solvent, chloroform : methanol: acetic acid (7: $3: 0.5)$ to obtain yellow powder $3-O-\beta-D-$ glucopyranoside $5.4 \mathrm{mg}$

\section{RESULT AND DISCUSSION}

The methanol extract of the dried powder $L$. microphyllum was concentrated and partitioned successively with n-hexane, ethyl acetate, and n-butanol. Part of n-hexane extract separated with various chromatographic techniques on the stationary phase silica gel and reverse phase to produce steroid compounds (1), (2) and the compound of Isoquercetin (3).

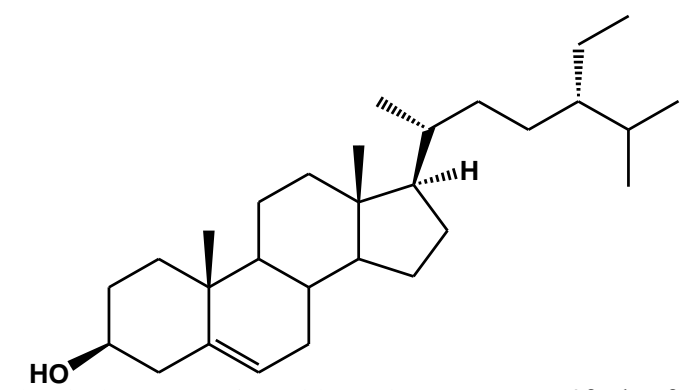

Fig. 1: Chemical structure of steroid (1) stigmast-5 (6)-en-3 $\beta$-ol or $\beta$-sitosterol.

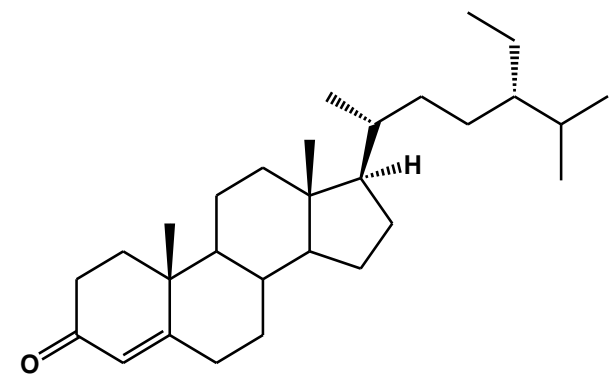

Fig. 2: Chemical structure of steroid (2)Stigmast-4-en-3-one.

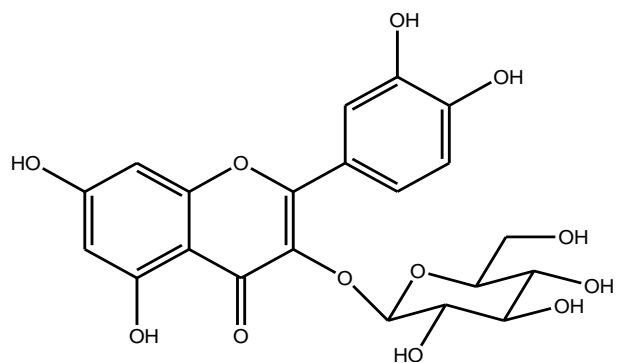

Fig. 3: Chemical structure of compound 3(3) 3-O- $\beta$-D-glucopyranoside or isoquercetin.

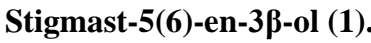

Crystal white needles; melting point $134-136{ }^{\circ} \mathrm{C}$. IR (KBr) $\mathrm{V}_{\text {Maks }} 3440,2934,2890,1640,1190 \mathrm{~cm}^{-1}$. ${ }^{1} \mathrm{H}$ NMR (asetond6, $500 \mathrm{MHz}$ ), Table 1; ${ }^{13} \mathrm{C}$ NMR (aseton-d6, $125 \mathrm{MHz}$ ), table 3, TOF MS (negatif ion mode) $[\mathrm{m} / \mathrm{z} 413.0911$, calculations for $\mathrm{C}_{28} \mathrm{H}_{50} \mathrm{O} \mathrm{m} / \mathrm{z}$ [414.0818].

${ }^{1} \mathrm{HNMR}$ (aseton-d6, $\left.500 \mathrm{MHz}\right) \delta 0.74(3 \mathrm{H}, \mathrm{s}), 0.84(3 \mathrm{H}$, d, 6.2) $0.86(3 \mathrm{H}, \mathrm{d}, 6.1), 0.88(3 \mathrm{H}, \mathrm{t}, 1.89), 0.95(1 \mathrm{H}, \mathrm{m}), 0.96(3 \mathrm{H}$, d, 6.7), $0.97(1 \mathrm{H}, \mathrm{m}), 1.02(3 \mathrm{H}, \mathrm{s}), 1.06(1 \mathrm{H}, \mathrm{dd}, 10.5 ; 5.5), 1.11$
(1H, dd, 10.5; 9.5), $1.13(1 \mathrm{H}, \mathrm{m}), 1.15(1 \mathrm{H}, \mathrm{m}), 1.15(1 \mathrm{H}, \mathrm{m}), 1.19$ $(2 \mathrm{H}, \mathrm{d}, 5.6), 1.22(1 \mathrm{H}, \mathrm{m}), 1.23(2 \mathrm{H}, \mathrm{m}), 1.27(2 \mathrm{H}, \mathrm{m}), 1.42(1 \mathrm{H}$, m), $1.55(2 \mathrm{H}, \mathrm{m}), 1.6(2 \mathrm{H}, \mathrm{m}), 1.69(1 \mathrm{H}, \mathrm{dt}, 9.5 ; 6.0), 1.77(1 \mathrm{H}, \mathrm{dt}$, $9.0 ; 6.0), 1.94$ (2H, dt, 5.6; 8.5), 1.95 (2H, m), 2.05 (2H, m), 2.21 (2H, d, 6.5), 3.39 (1H, m), $5.31(1 \mathrm{H}, \mathrm{d}, 5.6)$.

${ }^{13} \mathrm{CNMR}$ (aseton-d6, $\left.125 \mathrm{MHz}\right), \delta 37.4(\mathrm{t})(\mathrm{C}-1), 31.7(\mathrm{t})$ (C-2), 70.9 (d) (C-3), 42.5 (t) (C-4), 141.5 (s) (C-5), 120.7 (d) (c6), $31.8(\mathrm{t})(\mathrm{C}-7), 36.2$ (d) (C-8), 50.4 (d)(C-9), 36.5 (s) (C-10), 20.9 (t) (C-11), 39.8 (t) (C-12), 42.2 (s)(C-13), 56.8 (d)(C-14), $25.9(\mathrm{t})(\mathrm{C}-15), 28.1(\mathrm{t})(\mathrm{C}-16), 56.1(\mathrm{~d})(\mathrm{C}-17), 11.5$ (q)(C-18), 18.4 (q)(C-19), 40.5 (d) (C-20), 19.3 (q)(C-21), 33.9 (t)(C-22), 24.1 (t)(C-23), 45.9 (d)(C-24), 31.9 (d)(C-25), 18.9 (q) (C-26), 18.5 (q)(C-27), 22.9 (t)(C-28), 11.4 (q)(C-29).

These data were found to be consistent with those reported in the literature for stigmast-5 (6) -en-3 $\beta$-ol or $\beta$-sitosterol (Chaturvedula and Prakash, 2012), and compound 1 identified as $\beta$-sitosterol.

Table 1: NMR Data compound 1.

\begin{tabular}{|c|c|c|c|}
\hline \multirow{3}{*}{$\begin{array}{c} \\
\text { C } \\
\text { Position }\end{array}$} & \multirow{3}{*}{ 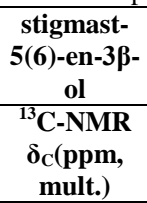 } & \multicolumn{2}{|c|}{ Compound 1} \\
\hline & & ${ }^{13} \mathrm{C}-\mathrm{NMR}$ & ${ }^{1} \mathrm{H}-\mathrm{NMR}$ \\
\hline & & $\delta_{\mathrm{C}}($ ppm, mult. $)$ & $\begin{array}{c}\delta_{\mathrm{H}}(\mathrm{ppm})(\Sigma \mathrm{H}, \text { mult., } J= \\
\mathrm{Hz})\end{array}$ \\
\hline \multirow[t]{2}{*}{1} & 37.5 & $37.4(\mathrm{t})$ & $1.06(1 \mathrm{H}, \mathrm{dd}, 10.5 ; 5.5)$ \\
\hline & & & $1.11(1 \mathrm{H}, \mathrm{dd}, 10.5 ; 9.5)$ \\
\hline \multirow[t]{2}{*}{2} & 31.9 & $31.7(\mathrm{t})$ & $1.69(1 \mathrm{H}, \mathrm{dt}, 9.5 ; 6.0)$ \\
\hline & & & $1.77(1 \mathrm{H}, \mathrm{dt}, 9.0 ; 6.0)$ \\
\hline 3 & 72.0 & 70.9 (d) & $3.39(1 \mathrm{H}, \mathrm{m})$ \\
\hline 4 & 42.5 & $42.5(\mathrm{t})$ & $2.21(2 \mathrm{H}, \mathrm{d}, 6.5)$ \\
\hline 5 & 140.9 & $141.5(\mathrm{~s})$ & - \\
\hline 6 & 121.9 & 120.7 (d) & $5.31(1 \mathrm{H}, \mathrm{d}, 5.6)$ \\
\hline 7 & 32.1 & $31.8(\mathrm{t})$ & $1.94(2 \mathrm{H}, \mathrm{dt}, 5.6 ; 8.5)$ \\
\hline 8 & 34.2 & 36.2 (d) & $1.42(1 \mathrm{H}, \mathrm{m})$ \\
\hline 9 & 50.3 & 50.4 (d) & $0.95(1 \mathrm{H}, \mathrm{m})$ \\
\hline 10 & 36.7 & $36.5(\mathrm{~s})$ & - \\
\hline 11 & 21.3 & $20.9(\mathrm{t})$ & $1.55(2 \mathrm{H}, \mathrm{m})$ \\
\hline 12 & 39.9 & $39.8(\mathrm{t})$ & $1.19(2 \mathrm{H}, \mathrm{d}, 5.6)$ \\
\hline 13 & 42.6 & $42.2(\mathrm{~s})$ & - \\
\hline 14 & 56.9 & $56.8(\mathrm{~d})$ & $1.13(1 \mathrm{H}, \mathrm{m})$ \\
\hline 15 & 26.3 & $25.9(\mathrm{t})$ & $1.23(2 \mathrm{H}, \mathrm{m})$ \\
\hline 16 & 28.5 & $28.1(\mathrm{t})$ & $1.95(2 \mathrm{H}, \mathrm{m})$ \\
\hline 17 & 56.3 & $56.1(\mathrm{~d})$ & $1.15(1 \mathrm{H}, \mathrm{m})$ \\
\hline 18 & 12.0 & $11.5(\mathrm{q})$ & $0.74(3 \mathrm{H}, \mathrm{s})$ \\
\hline 19 & 19.0 & $18.4(\mathrm{q})$ & $1.02(3 \mathrm{H}, \mathrm{s})$ \\
\hline 20 & 38.2 & 40.5 (d) & $1.22(1 \mathrm{H}, \mathrm{m})$ \\
\hline 21 & 19.2 & $19.3(q)$ & $0.86(3 \mathrm{H}, \mathrm{d}, 6.1)$ \\
\hline 22 & 34.2 & $33.9(\mathrm{t})$ & $2.05(2 \mathrm{H}, \mathrm{m})$ \\
\hline 23 & 26.1 & $24.1(\mathrm{t})$ & $1.6(2 \mathrm{H}, \mathrm{m})$ \\
\hline 24 & 46.1 & 45.9 (d) & $0.97(1 \mathrm{H}, \mathrm{m})$ \\
\hline 25 & 29.4 & 31.9 (d) & $1.15(1 \mathrm{H}, \mathrm{m})$ \\
\hline 26 & 20.1 & $18.9(\mathrm{q})$ & $0.84(3 \mathrm{H}, \mathrm{d}, 6.2)$ \\
\hline 27 & 19.6 & $18.5(\mathrm{q})$ & $0.96(3 \mathrm{H}, \mathrm{d}, 6.7)$ \\
\hline 28 & 23.3 & $22.9(\mathrm{t})$ & $1.27(2 \mathrm{H}, \mathrm{m})$ \\
\hline 29 & 12.2 & $11.4(\mathrm{q})$ & $0.88(3 \mathrm{H}, \mathrm{t}, 1.89)$ \\
\hline
\end{tabular}

*Measurements were taken at aseton- $d_{6}$ on $500 \mathrm{MHz}$ for ${ }^{1} \mathrm{H}$ and $125 \mathrm{MHz}$ for ${ }^{13} \mathrm{C}$.

\section{Stigmast-4-en-3-one (2),}

Crystal white needles; melting point $87-89^{\circ} \mathrm{C},{ }^{1} \mathrm{H}$ NMR (Pyridin-D6, $600 \mathrm{MHz}$ ), Table 2; ${ }^{13} \mathrm{C}$ NMR (Pyridin-D6, 150 Mhz), table 3. 
Table 2: NMR data compound 2.

\begin{tabular}{|c|c|c|c|c|}
\hline & \multicolumn{2}{|c|}{ Stigmast-4-en-3-one } & \multicolumn{2}{|c|}{ Compound 2} \\
\hline C position & $\begin{array}{c}{ }^{13} \mathrm{C}-\mathrm{NMR} \\
\delta_{\mathrm{C}}(\mathrm{ppm}, \text { mult. })\end{array}$ & $\begin{aligned} & { }^{1} \mathrm{H}-\mathrm{NMR} \\
\delta_{\mathrm{H}}(\mathrm{ppm}) & (\Sigma \mathrm{H}, \text { mult., } J=\mathrm{Hz})\end{aligned}$ & $\begin{array}{c}{ }^{13} \mathrm{C}-\mathrm{NMR} \\
\delta_{\mathrm{C}}(\mathrm{ppm}, \text { mult. })\end{array}$ & $\begin{aligned} & { }^{1} \mathrm{H}-\mathrm{NMR} \\
\delta_{\mathrm{H}}(\mathrm{ppm}) & (\Sigma \mathrm{\Sigma}, \text { mult., } J=\mathrm{Hz})\end{aligned}$ \\
\hline 1 & 35.9 & $1.48 ; 1.23$ & $35.7(\mathrm{t})$ & $\begin{array}{l}1.76(1 \mathrm{H}, \mathrm{m}) \\
1.44(1 \mathrm{H}, \mathrm{m})\end{array}$ \\
\hline 2 & 33.9 & $2.36 ; 2.31$ & $34.0(\mathrm{t})$ & $\begin{array}{l}2.32(1 \mathrm{H}, \mathrm{m}) \\
2.37(1 \mathrm{H}, \mathrm{m})\end{array}$ \\
\hline 3 & 199.6 & - & $199.3(\mathrm{~s})$ & - \\
\hline 4 & 123.7 & 5.70 & 123.9 (d) & $5.80(1 \mathrm{H}, \mathrm{s})$ \\
\hline 5 & 171.7 & - & $171.6(\mathrm{~s})$ & - \\
\hline 6 & 32.0 & $2.00 ; 1.90$ & $32.6(\mathrm{t})$ & $2.03(2 \mathrm{H}, \mathrm{m})$ \\
\hline 7 & 29.3 & $\begin{array}{c}1.17 ; 1.41 \\
-\end{array}$ & $32.1(\mathrm{t})$ & $\begin{array}{l}1.56(1 \mathrm{H}, \mathrm{m}) \\
1.61(1 \mathrm{H}, \mathrm{m})\end{array}$ \\
\hline 8 & 35.6 & 1.35 & $35.4(\mathrm{~d})$ & $1.76(1 \mathrm{H}, \mathrm{m})$ \\
\hline 9 & 53.8 & 1.32 & $53.7(d)$ & $0.68(1 \mathrm{H}, \mathrm{m})$ \\
\hline 10 & 39.6 & - & $38.5(\mathrm{~s})$ & - \\
\hline 11 & 21.0 & $1.54 ; 1.26$ & $20.9(\mathrm{t})$ & $1.32(2 \mathrm{H}, \mathrm{m})$ \\
\hline 12 & 39.6 & $1.55,1.31$ & $39.7(\mathrm{t})$ & $1.87(2 \mathrm{H}, \mathrm{m})$ \\
\hline 13 & 42.8 & & $42.4(\mathrm{~s})$ & - \\
\hline 14 & 55.9 & 1.02 & $55.8(\mathrm{~d})$ & $0.82(1 \mathrm{H}, \mathrm{m})$ \\
\hline 15 & 24.1 & $1.61 ; 1.33$ & $28.3(\mathrm{t})$ & $\begin{array}{l}1.62(1 \mathrm{H}, \mathrm{m}) \\
1.75(1 \mathrm{H}, \mathrm{m})\end{array}$ \\
\hline 16 & 33.8 & $1.61 ; 1.33$ & $24.2(\mathrm{t})$ & $1.45(2 \mathrm{H}, \mathrm{d}, 9.6)$ \\
\hline 17 & 55.8 & 1.15 & $56.0(\mathrm{~d})$ & $1.02(1 \mathrm{H}, \mathrm{m})$ \\
\hline 18 & 11.9 & 0.70 & $11.9(\mathrm{q})$ & $0.61(3 \mathrm{H}, \mathrm{s})$ \\
\hline 19 & 17.3 & 1.17 & $16.9(\mathrm{q})$ & $0.95(3 \mathrm{H}, \mathrm{s})$ \\
\hline 20 & 36.1 & 1.64 & $36.2(\mathrm{~d})$ & $1.31(1 \mathrm{H}, \mathrm{m})$ \\
\hline 21 & 18.6 & 0.97 & $18.8(\mathrm{q})$ & $0.79(3 \mathrm{H}$, d.6.6) \\
\hline 22 & 35.6 & $1.26 ; 1.26$ & $34.2(\mathrm{t})$ & $1.32(2 \mathrm{H}, \mathrm{m})$ \\
\hline 23 & 26.0 & $1.25 ; 1.25$ & $26.2(\mathrm{t})$ & $1.18(2 \mathrm{H}, \mathrm{m})$ \\
\hline 24 & 45.8 & 1.47 & $45.8(\mathrm{~d})$ & $1.19(2 \mathrm{H}, \mathrm{m})$ \\
\hline 25 & 28.1 & 1.83 & $29.3(\mathrm{~d})$ & $0.94(1 \mathrm{H}, \mathrm{m})$ \\
\hline 26 & 19.8 & 0.83 & $19.8(\mathrm{q})$ & $0.81(3 \mathrm{H}, \mathrm{d}, 6.5)$ \\
\hline 27 & 19.0 & 0.81 & $19.0(\mathrm{q})$ & $0.79(3 \mathrm{H}, \mathrm{d}, 6.7)$ \\
\hline 28 & 23.0 & $1.54 ; 1.54$ & $23.2(\mathrm{t})$ & $1.23(2 \mathrm{H}, \mathrm{m})$ \\
\hline 29 & 14.1 & 0.90 & $12.0(\mathrm{q})$ & $0.83(3 \mathrm{H}, \mathrm{t}, 6.5)$ \\
\hline
\end{tabular}

*400 MHz for ${ }^{1} \mathrm{H}$ and $100 \mathrm{MHz}$ for ${ }^{13} \mathrm{C}$, in $\mathrm{CDCl}_{3}$ (Halilu et al., 2013) and Measurements compound 3 were taken at $600 \mathrm{MHz}$ for ${ }^{1} \mathrm{H}$ and $150 \mathrm{MHz}$ for ${ }^{13} \mathrm{C}$, in Pyridin- $\mathrm{D}_{6}$

Table 3: NMR data compound 3.

\begin{tabular}{|c|c|c|c|c|}
\hline \multirow[b]{2}{*}{ C Position } & \multicolumn{2}{|c|}{ 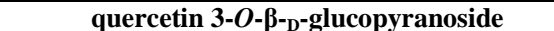 } & \multicolumn{2}{|c|}{ Compound 3} \\
\hline & $\begin{array}{l}{ }^{13} \mathrm{C} \mathrm{NMR} \\
\delta \mathrm{c} \text { (mult) }\end{array}$ & $\begin{array}{c}{ }^{1} \mathrm{H} \text { NMR } \delta_{\mathrm{H}} \\
\text { (Integral, mult, } \boldsymbol{J}=\mathrm{Hz} \text { ) }\end{array}$ & ${ }^{13} \mathrm{C}$ NMR $\delta \mathrm{c}$ (mult) & $\begin{array}{c}{ }^{1} \mathrm{H} \text { NMR } \delta_{\mathrm{H}} \\
\text { (Integral, mult, } \boldsymbol{J}=\mathrm{Hz} \text { ) }\end{array}$ \\
\hline 2 & $158.44(\mathrm{~s})$ & - & $156.97(\mathrm{~s})$ & - \\
\hline 3 & $135.64(\mathrm{~s})$ & - & $134.09(\mathrm{~s})$ & - \\
\hline 4 & $179.48(\mathrm{~s})$ & - & $178.96(\mathrm{~s})$ & - \\
\hline 5 & $162.99(\mathrm{~s})$ & - & $161.36(\mathrm{~s})$ & - \\
\hline 6 & 99.89 (d) & $6.19(1 \mathrm{H}, \mathrm{d}, 2)$ & $100.38(d)$ & - \\
\hline 7 & $165.97(\mathrm{~s})$ & - & $163.15(\mathrm{~s})$ & $6.07(1 \mathrm{H}, \mathrm{d}, 2.4)$ \\
\hline 8 & 94.73 (d) & $6.38(1 \mathrm{H}, \mathrm{d}, 2)$ & 94.67 (d) & - \\
\hline 9 & $158.44(\mathrm{~s})$ & - & $157.60(\mathrm{~s})$ & $6.23(1 \mathrm{H}, \mathrm{d}, 2.4)$ \\
\hline 10 & $105.68(\mathrm{~s})$ & - & $102.68(\mathrm{~s})$ & - \\
\hline 1 ' & $123.08(\mathrm{~s})$ & - & $121.61(\mathrm{~s})$ & - \\
\hline 2 ' & $117.59(\mathrm{~d})$ & $7.71(1 \mathrm{H}, \mathrm{d}, 2)$ & $116.00(d)$ & - \\
\hline 3 , & $145.87(\mathrm{~s})$ & - & $144.73(\mathrm{~s})$ & $7.68(1 \mathrm{H}, \mathrm{d}, 2.4)$ \\
\hline 4 ' & $149.83(\mathrm{~s})$ & - & $148.81(\mathrm{~s})$ & - \\
\hline 5 , & $116.01(d)$ & $6.87(1 \mathrm{H}, \mathrm{d}, 8.4)$ & $114.67(d)$ & - \\
\hline 6 ' & $123.20(\mathrm{~d})$ & $7.58(1 \mathrm{H}, \mathrm{dd}, 2 ; 8.4)$ & $121.75(\mathrm{~d})$ & $6.82(1 \mathrm{H}, \mathrm{d}, 8.4)$ \\
\hline 1 ", & $104.39(\mathrm{~d})$ & $5.23(1 \mathrm{H}, \mathrm{d}, 7.6)$ & $103.54(d)$ & $7.55(1 \mathrm{H}, \mathrm{dd}, 2.4 ; 8.4)$ \\
\hline 2 "' & 75.73 (d) & $3.48(1 \mathrm{H}, \mathrm{d}, 9.2)$ & $74.35(\mathrm{~d})$ & $5.11(1 \mathrm{H}, \mathrm{dd}, 6.8 ; 7.2)$ \\
\hline 3"' & 78.11 (d) & $3.35(1 \mathrm{H}, \mathrm{d}, 8.8)$ & $76.81(\mathrm{~d})$ & $3.46(1 \mathrm{H}, \mathrm{d}, 9)$ \\
\hline $4 "$, & 71.22 (d) & $3.43(1 \mathrm{H}, \mathrm{d}, 9.6)$ & 69.78 (d) & $3.39(1 \mathrm{H}, \mathrm{d}, 9.6)$ \\
\hline 5, & 78.35 (d) & $3.24(1 \mathrm{H}, \mathrm{m})$ & $77.00(\mathrm{~d})$ & $3.32(1 \mathrm{H}, \mathrm{d}, 9.6)$ \\
\hline \multirow[t]{3}{*}{$6 "$} & $62.58(\mathrm{t})$ & $3.73(1 \mathrm{H}, \mathrm{dd}, 2 ; 11.6)$ & $61.14(\mathrm{t})$ & $3.19(1 \mathrm{H}, \mathrm{m})$ \\
\hline & & $3.56(1 \mathrm{H}, \mathrm{dd}, 5.2 ; 11.6)$ & & $3.68(1 \mathrm{H}, \mathrm{dd}, 2.4 ; 11.4)$ \\
\hline & & & & $3.56(1 \mathrm{H}, \mathrm{dd}, 6.0 ; 11.4)$ \\
\hline
\end{tabular}

* $400 \mathrm{MHz}$ for ${ }^{1} \mathrm{H}$ and $100 \mathrm{MHz}$ for ${ }_{13} \mathrm{C}$, in $\mathrm{CD}_{3} \mathrm{OD}$ (Islam et al., 2012) and Measurements compound 3 were taken at $600 \mathrm{MHz}$ for ${ }^{1} \mathrm{H}$ and $150 \mathrm{MHz}$ for ${ }^{13} \mathrm{C}$. 
${ }^{1} \mathrm{H}$ NMR (Pyridin-D6, $\left.600 \mathrm{MHz}\right) \delta 0.61(3 \mathrm{H}, \mathrm{s}), 0.68$ $(1 \mathrm{H}, \mathrm{m}), 0.79$ (3H, d.6.6), 0.79 (3H, d, 6.7), $0.81(3 \mathrm{H}, \mathrm{d}, 6.5), 0.82$ $(1 \mathrm{H}, \mathrm{m}), 0.83(3 \mathrm{H}, \mathrm{t}, 6.5), 0.94(1 \mathrm{H}, \mathrm{m}), 0.95(3 \mathrm{H}, \mathrm{s}), 1.02(1 \mathrm{H}$, $\mathrm{m}), 1.18(2 \mathrm{H}, \mathrm{m}), 1.19(2 \mathrm{H}, \mathrm{m}), 1.23(2 \mathrm{H}, \mathrm{m}), 1.31(1 \mathrm{H}, \mathrm{m}), 1.32$ $(2 \mathrm{H}, \mathrm{m}) 1.32(2 \mathrm{H}, \mathrm{m}), 1.44(1 \mathrm{H}, \mathrm{m}), 1.45(2 \mathrm{H}, \mathrm{d}, 9.6), 1.56(1 \mathrm{H}$, $\mathrm{m}), 1.61(1 \mathrm{H}, \mathrm{m}), 1.62(1 \mathrm{H}, \mathrm{m}), 1.75(1 \mathrm{H}, \mathrm{m}), 1.76(1 \mathrm{H}, \mathrm{m}), 1.76$ $(1 \mathrm{H}, \mathrm{m}), 1.87(2 \mathrm{H}, \mathrm{m}), 2.03(2 \mathrm{H}, \mathrm{m}), 2.32(1 \mathrm{H}, \mathrm{m}), 2.37(1 \mathrm{H}, \mathrm{m})$, $5.80(1 \mathrm{H}, \mathrm{s})$.

${ }^{13} \mathrm{C}$ NMR (Pyridin-D6, $\left.150 \mathrm{Mhz}\right) \delta 35.7(\mathrm{t})(\mathrm{C}-1), 34.0(\mathrm{t})$ (C-2), 199.3 (s) (C-3), 123.9 (d) (C-4), 171.6 (s) (C-5), 32.6 (t) (C6), 32.1(t)(C-7), 35.4 (d) (C-8), 53.7 (d)(C-9), 38.5 (s)(C-10), 20.9 (t) (C-11), 39.7 (t) (C-12), 42.4 (s) (C-13), 55.8 (d) (C-14), 28.3 (t) (C-15), 24.2 (t) (C-16), 56.0 (d) (C-17), 11.9 (q) (C-18), 16.9 (q) (C-19), 36.2 (d) (C-20), 18.8 (q) (C-21), 34.2 (t) (C-22), 26.2 (t) (C-23), 29.3 (d) (C-24), 45.8 (d) (C-25), 19.8 (q) (C-26), 19.0 (q) (C-27), 23.2 (t) (C-28), 12.0 (q) (C-29). These data were found to be consistent with those reported in the literature for Stigmast-4en-3-one (Halilu et al, 2013; Barla et al, 2006 and DellaGreca et $a l, 1990)$, and compound 2 identified as Stigmast-4-en-3-one.

\section{3-O-p-D-glucopyranoside (3)}

Yellow powder; melting point $872.6{ }^{\circ} \mathrm{C} .{ }^{1} \mathrm{H}$ NMR (Pyridin-D6, $600 \mathrm{MHz}$ ), table $3 ;{ }^{13} \mathrm{C}$ NMR (Pyridin-D6, $150 \mathrm{Mhz}$ ), table 3.

${ }^{1} \mathrm{H}$ NMR (Pyridin-D6, $\left.600 \mathrm{MHz}\right) \delta 6.07(1 \mathrm{H}, \mathrm{d}, 2.4)$, $6.23(1 \mathrm{H}, \mathrm{d}, 2.4), 7.68(1 \mathrm{H}, \mathrm{d}, 2.4), 6.82(1 \mathrm{H}, \mathrm{d}, 8.4), 7.55(1 \mathrm{H}, \mathrm{dd}$, $2.4 ; 8.4), 5.11(1 \mathrm{H}, \mathrm{dd}, 6.8 ; 7.2), 3.46(1 \mathrm{H}, \mathrm{d}, 9), 3.39(1 \mathrm{H}, \mathrm{d}, 9.6)$, $3.32(1 \mathrm{H}, \mathrm{d}, 9.6), 3.19(1 \mathrm{H}, \mathrm{m}), 3.68(1 \mathrm{H}, \mathrm{dd}, 2.4 ; 11.4), 3.56(1 \mathrm{H}$, $\mathrm{dd}, 6.0 ; 11.4)$.

${ }^{13} \mathrm{C}$ NMR (Pyridin-D6, $\left.150 \mathrm{Mhz}\right) \delta 156.97$ (s) $(\mathrm{C}-2)$, 134.09 (s) (C-3), 178.96 (s) (C-4), 161.36 (s) (C-5), 100.38 (d) (C6), 163.15 (s) (C-7), 94.67 (d) (C-8), 157.60 (s) (C-9), 102.68 (s) (C-10), 121.61 (s) (C-1'), 116.00 (d) (C-2'), 144.73 (s) (C-3'), 148.81 (s) (C-4'), 114.67 (d) (C-5'), 121.75 (d) (C-6'), 103.54 (d) (C-1'), 74.35 (d) (C-2'), 76.81 (d) (C-3') 69.78 (d) (C-4'), 77.00 (d) (C-5"), 61.14 (t) (C-6").

These data were found to be consistent with those reported in the literature for 3-O- $\beta$-D-glucopyranoside (Shi et al, 2016; Islam et al, 2012 and Li et al., 2008), and compound 3 identified as 3-O- $\beta$-D-glucopyranoside.

Compound 1 obtained in the form of colorless white crystal with a melting point of $134-136^{\circ} \mathrm{C}$ and soluble in acetone. The molecular formula of compound 1 was established as $\mathrm{C}_{29} \mathrm{H}_{50} \mathrm{O}$ based HR-TOFMS spectrum (negative ion mode) $\mathrm{m} / \mathrm{z} 413.0911$, the calculation for $\mathrm{C}_{29} \mathrm{H}_{50} \mathrm{O} \mathrm{m} / \mathrm{z} 414.0818$ with NMR data (Table 1 ), therefore required five double bond equivalent. Compound 1 does not fluoresce under ultraviolet light at a wavelength of 254 and $360 \mathrm{~nm}$, indicating the absence of conjugated double bonds. Infrared absorption due to a hydroxyl group, a double bond, and the ether was observed in $3440,2934,2890,1640,1190 \mathrm{~cm}^{-1} .{ }^{1} \mathrm{H}$ NMR spectrum compounds 1 expressed their olefinic proton signals $\left[\delta_{\mathrm{H}} 5.31(1 \mathrm{H}, \mathrm{d}, J=4.9 \mathrm{~Hz})\right.$, six methyl proton signal consisting of two methyl tertiary $\left[\delta_{\mathrm{H}} 0.74(3 \mathrm{H}, \mathrm{s})\right.$ and $1.02(3 \mathrm{H}$, s)], four secondary methyl $\left[\delta_{\mathrm{H}} 0.84(3 \mathrm{H}, \mathrm{d}, J=6.2 \mathrm{~Hz}), 0.86(3 \mathrm{H}, \mathrm{d}\right.$,
$J=6.1 \mathrm{~Hz}), 0.88(3 \mathrm{H}, \mathrm{t}, J=1.89 \mathrm{~Hz})$ dan $0.96(3 \mathrm{H}, \mathrm{d}, J=6.7 \mathrm{~Hz})]$ and one metyhn signal sp $\mathrm{sp}^{3}$ oxygenated on $3.39(1 \mathrm{H}, \mathrm{m})$. Total carbon signals twenty-nine observed in ${ }^{13} \mathrm{C}$ NMR spectrum. These signals are specified with DEPT and HMQC experiment as two carbon $\mathrm{sp}^{2}$ $\left(\delta_{\mathrm{C}} 120.7\right.$ and 141.5), six methyls, eleven methylene $\mathrm{sp}^{3}$, seven methyn $\mathrm{sp}^{3}$, one methyn oxygenated with $\delta_{\mathrm{C}} 70.9$ and two carbon $\mathrm{sp}^{3}$ quartener. This functionality is counted as one of the totals of five double bond equivalent. Comparison of physical data and physicochemical compound $\mathbf{1}$ with stigmast-5(6)-en-3 $\beta$-ol (Chaturvedula and Prakash, 2012) shows a very high suitability, thus compound 1 was identified as stigmast-5(6)-en-3 $\beta$-ol or $\beta$ sitosterol.

The reported of $\beta$-phytosterol compounds in plants $L$. microphyllum first reported in this study thus provide clues phytochemical their steroid compounds in the genus Lygodium.

Compound 2 obtained in the form of a colorless white crystals with a melting point $87-89^{\circ} \mathrm{Cand}$ soluble in chloroform. $1 \mathrm{H}$ NMR spectrum compounds show six methyl signal at $\delta_{\mathrm{H}} \mathrm{ppm} 0.61$ $(3 \mathrm{H}), 0.79(3 \mathrm{H}), 0.79(3 \mathrm{H}), 0.81(3 \mathrm{H}), 0.83(3 \mathrm{H})$, and $0.95(3 \mathrm{H})$, these suggest that there are six methyl group. The presence of down field signal at $\delta_{\mathrm{H}} \mathrm{ppm} 5.80(1 \mathrm{H})$ shows olefinic proton. ${ }^{13} \mathrm{C}$ NMR spectrum show 29 signal (Table 2) suggesting that compound consist of 29 carbon atom. Compound 2 does not fluorescent under ultraviolet light at a wavelength 254 and 360 $\mathrm{nm}$, indicated the absence of conjugated double bonds. Comparison of physical data and physicochemical compound 2 Stigmast-4-en-3-one shows a very high suitability (Halilu et al, 2013; Barla et al, 2006 and Della Greca et al, 1990), thus compound 2 was identified as Stigmast-4-en-3-one.

Compound 3 obtained in the form of yellow powder with a melting point $872.6{ }^{\circ} \mathrm{C}$ and completely soluble in methanol, Fluorescent under ultraviolet light at a wavelength 254 and 360 $\mathrm{nm}$, indicated there is founded conjugated double bonds. Comparison of physical data and physicochemical compound3.3O- $\beta$-D-glucopyranoside or Isoquercetin shows a very high suitability (Shi et al, 2016; Islam et al, 2012 and Li et al, 2008), thus compound 3 was identified as 3-O- $\beta$-D-glucopyranoside or Isoquercetin.

\section{CONCLUSION}

Two steroids, stigma-5(6)-en-3 $\beta$-ol or $\beta$-sitosterol (1) and Stigmast-4-en-3-one (2) and quercetin 3-O- $\beta$-D-glucopyranoside or Isoquercetin (3) in plants L. microphyllum first reported in this study.

\section{ACKNOWNLEDMENT}

Authors gratefully acknowledge to Directorate General of Higher Education, Ministry of Research, Technology and higher education, Indonesia on funds Competitive Research Grant and PKPI Sandwich Like 2015 Scholarship. Our thanks also to Prof. Dr. Unang Supratman in Central Laboratory, Padjadjaran University, Mr. Ahmad Darmawan, M.Sc. and Sofa Fajriah, M.Si in Chemical Research Center, Indonesian Institute of Sciences, 
Serpong, Tangerang and Prof. Dr. Yoshito Shiono in Laboratory of Natural Products, Department Of Bioresources Engineering, Faculty of Agriculture, Yamagata University, Japan for NMR measurements.

\section{REFERENCES}

Barla A, Bİrman H, KÜLTÜR Ş, and Öksüz S. Secondary metabolites from Euphorbia helioscopia and their vasodepressor activity. Turkish Journal of Chemistry. 2006; 30(3): 325-332. Available at: http://journals.tubitak.gov.tr/chem/abstract.htm?id=8338.

Cambie RC \& Ash J., 1994. Fijian Medicinal Plants. CSIRO. Melbourne.

Chaturvedula VSR \& Prakash I. Isolation of stigmasterol and $\beta$ sitosterol from the dichloromethane extract of Rubus suavissimus. International Current Pharmaceutical Journal. 2012; 1(9): 239-242. [ONLINE] Available http://imsear.hellis.org/handle/123456789/152876

Chen L, Zhang G, He J, Jin Guan J, Pan C, Mi W, \& Wang Q. New naphthoquinone from the root of Lygodium japonicum (Thunb.) Sw. Journal of Natural Medicine.2010; 64: 114-116. doi: 10.1007/s11418009-0376-y

Della Greca M, Monaco P, \& Previtera L. Stigmasterols from Typha latifolia. Journal of Natural Products.1990; 53(6): 1430-1435. DOI: $10.1021 / \mathrm{np50072a005.}$

Duan Y, Dai, He YR, Kurihara H, Li Y, \& Yao X. A new phenylpropanoid glucoside from the aerial parts of Lygodium japonicum. Journal of Asian Natural Products Research. 2012; 14 (3): 286-292. doi:10.1080/10286020.2011.650690

Guo-Gang Z, Ying-Cui H, Hong-Xia L, Lin-Xia Z, \& Li-Juan C. 2012. The Research of Lygodium. Intechopen. Shanghai.

Halilu ME, October N, Balogun M, Agunu A, Abubakar A. \& Abubakar MS. Isolation and Characterization of Steroids from Petroleum Ether Extract of Stem Bark of Parinari curatellifolia Planch ex. Benth (Chrysobalanaceae). Journal of Natural Sciences Research. 2013; 3(6).

Islam M, Al-Amin MD, Siddiqi MMA, Akter S, Haque MM, Sultana N, \& Chowdhury AS, Isolation of quercetin-3-O-beta-Dglucopyranoside from the leaves of Azadirachta Indica and antimicrobial and cytotoxic screening of the crude extracts. Dhaka University journal of science. 2012; 60(1):11-14.
Karthik V, Raju K, Ayyanar M, Gowrishankar K, \& Sekar T. Ethnomedicinal uses of pteridophytes in Kolli Hills, Eastern Ghats of Tamil Nadu, India. Journal of Natural Products Plant Resources. 2011; $50-55$

Lee S, Xiao C, \& Pei S. Ethnobotanical survey of medicinal plants at periodic markets of Honghe Prefecture in Yunnan Province, China. Journal of Ethnopharmacology. 2008; 117: 362-377. doi:10.1016/j.jep.2008.02.001

Li YL, Li J, Wang NL,\& Yao XS. Flavonoids and a new polyacetylene from Bidens parvifloraWilld. Molecules. 2008; 13(8): 19311941. doi:10.3390/molecules13081931.

Shi GQ, Yang J, Liu J, Liu SN, Song HX, Zhao WE, \& Liu YQ. Isolation of flavonoids from onion skins and their effects on K562 cell viability. Bangladesh Journal of Pharmacology, 2016; 11(S1),: 18-25. DOI: http://dx.doi.org/10.3329/bjp.v11iS1.26419.

Upreti K, Jalal JS, Tewari LM, Joshi GC, Pangtey YPS, \& Tewari G. Ethnomedicinal uses of Pteridophytes of Kumaun Himalaya, Uttarakhand, India. Journal of American Science. 2009; 5: 167-170.

Wallace RA, Sander GP, \& Ferl RJ. 1991. Biology: The Science of Life. Harper Collins. New York.

Ye W, Fan C, Zhang L, Yin Z, \& Zhao S. A new phenolic glycoside from the roots of Lygodium japonicum. Fitoterapia. 2007; 78: 600-601. doi:10.1016/j.fitote.2007.04.003

Zhang L, Yin Z, Ye W. Flavonoids from Lygodium japonicum. Biochemical Systematics and Ecology. 2006; 34: 885-886. doi: 10.1016/j.bse.2006.04.009

Zeng-fu LI, Huil H, Hang-yi Z, \& Jun-chen Z. Review on the extraction of flavonoids from fern. Journal of San University. 2008; 25: 22.

Zheng XL, \& Xing FW. Ethnobotanical study on medicinal plants around Mt. Yinggeling, Hainan Island, China. Journal of Ethnopharmacology. 2009; 124: 197-210. doi:10.1016/j.jep.2009.04.042

Zhu L, Zhang G, Chen L, Wang S, Li P, Li L. A new ecdysteroside from Lygodium japonicum (Thunb.) Sw. Journal of Natural Medicinal. 2009; 63: 215-219. doi:10.1007/s11418-008-0310-8.

\section{How to cite this article:}

Kuncoro H, Farabi K, Rijai L. Steroids and isoquercetin From Lygodium microphyllum. J App Pharm Sci, 2017; 7 (11): 136-141. 